\title{
Monitoramento de tempo e temperatura de distribuição de preparações à base de carne em escolas municipais de Natal (RN), Brasil ${ }^{1}$
}

\author{
M onitoring exposure time and distribution \\ temperature of meat-based meals served in \\ municipal schools in Natal, Brazil
}

Monique Silveira ROSA²

Sarah Regina Ferreira de NEGREIROS ${ }^{3}$

Larissa Mont'Alverne Jucá SEABRA ${ }^{3}$

Tânia Lúcia Montenegro STAM FORD ${ }^{4}$

RE S U M O

\section{Objetivo}

Monitorar o tempo e a temperatura de distribuição de preparações à base de carne servidas em escolas municipais de Natal (RN).

\section{Métodos}

Foram selecionadas 27 escolas da rede municipal de ensino, de forma aleatória, divididas em estratos por diferentes regiões administrativas. As medições de temperatura de preparações à base de carne foram verificadas quatro vezes em cada escola e o tempo de exposição destas foi verificado ao final da cocção e no início e no final da distribuição, com um termômetro digital do tipo espeto e relógio digital. Os resultados foram comparados com os padrões da resolução da diretoria colegiada $n^{\circ} 216 / 2004$, da Agência Nacional de Vigilância Sanitária do Ministério da Saúde.

\section{Resultados}

Para temperatura de cocção, $100 \%$ das escolas apresentaram-se dentro dos padrões (acima de $70^{\circ} \mathrm{C}$ ). Entretanto, no início e no final da distribuição, 100\% das escolas das Regiões Leste e Sul apresentaram

\footnotetext{
${ }^{1}$ Artigo elaborado a partir da dissertação de M.S.ROSA, intitulada "Avaliação das condições higiênico-sanitárias da produção de refeições à base de carne da al imentação escolar no município de Natal, RN". Programa de Pós-Graduação em Ciências da Saúde, Universidade Federal do Rio Grande do Norte.

2 Universidade Federal do Rio Grande do Norte, Centro de Ciências da Saúde, Programa de Pós-Graduação em Ciências da Saúde. Natal, RN, Brasil.

${ }^{3}$ Universidade Federal do Rio Grande do Norte, Centro de Ciências da Saúde, Departamento de Nutrição. Natal, RN, Brasil.

4 Universidade Federal de Pernambuco, Centro de Ciências da Saúde, Departamento de Nutrição. Av. Prof. M oraes Rego, 1235, Cidade Universitária, 50670-901, Recife, PE, Brasil. Correspondência para/Correspondence to: T.L.M.STAMFORD. E-mail: ४lmstamford@yahoo.com.br>.
} 
temperaturas inadequadas (abaixo de $60^{\circ} \mathrm{C}$ ). Na Região Oeste $70 \%$ das escolas apresentaram temperaturas em desacordo no início da distribuição e $90 \%$ no final dessa etapa. Na Região Norte verificou-se que $91 \%$ das escolas apresentaram temperaturas impróprias no início e $82 \%$ no final da distribuição. As médias do tempo de espera das preparações foram de 59, 49, 66 e 48 minutos para as regiões Leste, Oeste, Norte e Sul, respectivamente.

\section{Conclusão}

Há necessidade da adoção de Boas Práticas nas escolas municipais de Natal, a fim de uma manutenção efetiva da temperatura de distribuição das refeições no intuito de garantir uma alimentação de qualidade sanitária satisfatória aos escolares, evitando-se as intoxicações alimentares decorrentes da ineficácia das temperaturas.

Termos de indexação: Alimentação escolar. Carne. Higiene dos alimentos.

\section{A B S T R A C T}

\section{Objective}

To monitor exposure time and temperature of meat-based meals served in municipal schools in Natal, Brazil.

\section{Methods}

Twenty-seven municipal schools w ere randomly selected and stratified by administrative region. The temperature of meat-based meals was verified four times at each school and exposure time was checked at the end of cooking and at initial and final distribution, with a digital thermometer. The results were compared with the standards of Agência Nacional de Vigilância Sanitária of the Ministério da Saúde, resolution n²16/2004.

\section{Results}

With respect to cooking temperature, $100 \%$ of the schools were within the standards (above $70 \circ \mathrm{C}$ ). How ever, both at initial and final distribution, $100 \%$ of the schools from the Eastern and Southern regions exhibited inadequate temperatures (below 60 을. In the Western region $70 \%$ of the schools had different temperatures at the outset of distribution and $90 \%$ at the conclusion. In the northern region it was verified that $91 \%$ and $82 \%$ of the schools had inappropriate initial and final temperatures, respectively. Mean waiting time for the meals was 59, 49, 66, and 48 minutes for the Eastern, Western, Northern and Southern regions, respectively.

\section{Conclusion}

Good practices must be adopted in the municipal schools of Natal, in order to effectively maintain the distribution temperatures of meals, thus ensuring satisfactory sanitary quality of the food served to schoolchildren and avoiding food intoxication occasioned by improper temperatures.

Indexing terms: School feeding. Meat. Food hygiene.

\section{N T RO D U Ç Ã O}

O Programa Nacional de Alimentação Escolar (PNAE) é o programa que prevê a transferência de recursos federais para Estados, M unicípios e o Distrito Federal, com a finalidade de adquirir os alimentos para a merenda escolar, em caráter suplementar ${ }^{1}$. O PNAE apresenta como diretrizes o emprego da alimentação saudável e a aplicação da educação alimentar e nutricional ${ }^{2}$, tendo como objetivo assegurar que sejam supridas, parcialmente, as necessidades nutricionais dos escolares da pré-escola e do ensino fundamental das redes públicas e filantrópicas de ensino, além de estimular a integração de temas relativos à nutrição ao currículo escolar ${ }^{3}$.

Com a implementação do processo de descentralização, em 1993, ocorre o estímulo da participação popular no conjunto das ações de gestão do Programa, esperando a regularidade do fornecimento da merenda, a melhoria da qualidade das refeições, o atendimento dos hábitos alimentares, a diversificação da oferta de alimentos, o incentivo à economia local e regional e a diminuição dos custos operacionais ${ }^{4}$.

A aquisição dos alimentos para o PNAE deve obedecer ao cardápio planejado pelo 
nutricionista e ser realizada, prioritariamente, no município, no estado, no Distrito Federal ou nas regiões de destino, visando à redução dos custos e ao atendimento das diretrizes do Programa ${ }^{5}$. Os cardápios devem ter um equilíbrio em macronutrientes: carboidratos, lipídeos e proteínas de alto valor biológico, sendo, então, de fundamental importância a introdução de preparações à base de carne na alimentação escolar, devido ao seu excelente valor nutricional ${ }^{6}$.

Para Philippi', carne é o conjunto de tecidos de cor e consistência características que recobre o esqueleto dos animais. Segundo Franco $\&$ Landgraf ${ }^{8}$, a carne apresenta uma composição química que a torna excelente meio de cultura, pois é um alimento rico em substâncias nitrogenadas, minerais e vitaminas, além disso, o pH e a atividade água são favoráveis para o desenvolvimento da maioria dos microorganismos. Essas características contribuem para que a carne seja um dos alimentos mais implicados em surtos de doenças transmitidas por alimentos (DTAs).

A Organização Mundial de Saúde afirma que a alta temperatura contribui para a garantia da inocuidade dos alimentos, podendo eliminar quase todos os microorganismos patogênicos9. Portanto, o fator temperatura e a possibilidade de diminuição de tempos e de técnicas de manipulação e processamento de alimentos são meios que podem ser utilizados eficientemente no combate a microorganismos ${ }^{10}$. É muito importante 0 monitoramento desses fatores, principalmente nas escolas, por serem estabelecimentos comumente passíveis de surto ${ }^{11}$, por fornecerem alimentos a um grande número de crianças e por estas possuírem o sistema imunológico mais susceptível às DTAs.

Nas escolas, as refeições são preparadas em grande quantidade e até 0 momento da distribuição, em sua maioria, permanecem expostas à temperatura ambiente. Reconhecendo os riscos que estas práticas oferecem à saúde, especialmente das crianças, e a importância social do fornecimento de refeições nas escolas, este trabalho teve por objetivo monitorar o tempo e a temperatura de distribuição das preparações à base de carne nas escolas públicas municipais de Natal (RN), para averiguar se estas estão sendo preparadas e distribuídas de acordo com os padrões vigentes da resolução da diretoria colegiada (RDC) № 216, de 15 de setembro de 2004, da Agência Nacional de Vigilância Sanitária $(\text { ANVISA })^{12}$.

\section{MÉTO DOS}

O M unicípio de Natal apresenta 70 escolas, distribuídas em quatro regiões administrativas. Para o plano amostral, optou-se por uma amostra aleatória estratificada proporcional ${ }^{13}$, sendo os respectivos estratos definidos pelas diferentes regiões. Desse modo foram analisadas 27 escolas da rede municipal de educação da cidade de Natal (RN): 3 na Região Sul, 11 na Região Norte, 10 na Região Oeste e 3 na Região Leste.

Foram medidas as temperaturas das preparações prontas à base de carne ao final da cocção, no início e no final da distribuição, assim como foi verificado o tempo de exposição das preparações entre o final da cocção e o final da distribuição, no período de março a setembro de 2005. Utilizaram-se um termômetro digital do tipo espeto com variação de $-30 \mathrm{a}+180^{\circ} \mathrm{C}$ e um relógio digital. 0 tempo para a leitura de temperatura do termômetro foi de 1 minuto. Antes e após cada medição foi realizada a higienização do termômetro, utilizando papel toalha não reciclável e álcool a $70 \%$.

Em cada escola foram monitorados o tempo e a temperatura de quatro preparações à base de carne, realizadas em dias e/ou turnos diferentes. Como foram pesquisadas 27 escolas com quatro mensurações em cada uma delas, verificaram-se o tempo e a temperatura de 108 preparações à base de carne. Destas quatro mensurações foi calculada a média das temperaturas de cada escola, tendo-se obtido o percentual de inadequação das mesmas ao final da cocção, ao início e ao final da distribuição de preparações à 
base de carne das escolas municipais de Natal (RN), nas diferentes regiões administrativas.

Os padrões de temperatura das preparações à base de carne adotados foram os preconizados pela RDC $N^{\circ} 216$, de 15 de setembro de 2004 da ANVISA ${ }^{11}$ : maior que $70^{\circ} \mathrm{C}$ ao final da cocção; maior que $60^{\circ} \mathrm{C}$ para o início e o final da distribuição, por no máximo 6 horas.

Foram verificadas também as condições de exposição das preparações à base de carne durante 0 tempo de espera, por meio de observação direta.

Foi realizada estatística descritiva dos dados, utilizando o software Statistica 6.014.

\section{RESULTADOSE DISCUSSÃ O}

Pode-se observar (Tabela 1) que a temperatura ao final da cocção estava de acordo com os padrões vigentes, já que o tratamento térmico garantiu que todas as partes dos alimentos atingissem temperaturas iguais ou superio res a $70^{\circ} \mathrm{C}$, como preconiza a resolução RDC $216^{12}$. Provavelmente, a temperatura empregada durante a cocção das preparações monitoradas, foi eficiente para impedir a sobrevivência de células vegetativas de bactérias que causam DTAs.

Outros trabalhos monitoraram a temperatura de preparações servidas em escolas. Oliveira et al. ${ }^{15}$, avaliando cinco preparações à base de carne servidas no Programa de Alimentação
Escolar de um Município da Grande São Paulo, observaram que três preparações apresentaram temperaturas de cocção inadequadas.

A temperatura de cocção é um fator fundamental no controle das condições sanitárias do alimento, como demonstram alguns autores. Fortuna ${ }^{16}$ analisou 22 amostras de carne bovina, que seriam utilizadas na merenda de escolas municipais e estaduais antes e após a cocção, e verificou que apenas duas amostras de carne crua $(9,1 \%)$ encontravam-se acima dos padrões para Escherichia coli e Coliformes fecais, porém a cocção adequada suplantou essa contaminação. Vieira et al. ${ }^{17}$, analisando a qualidade microbiológica da carne in natura e de pratos prontos à base de carne servida, em nove escolas estaduais de Poços de Caldas (MG), observaram que a cocção levou a uma redução considerável dos mesófilos aeróbiose também eliminou E. colie Staphylococcus coagulase positiva, presentes nas amostras investigadas.

Os valores médios de temperatura ao início e ao final da distribuição encontram-se, também, na Tabela 1. Foi observado, durante a coleta dos dados, que no período de pós-cocção até o início e o final da distribuição, as preparações permaneceram expostas à temperatura ambiente. Ao observar a média das temperaturas de distribuição, verifica-se uma redução de temperatura, que ocorreu devido à inexistência de sistema de manutenção de calor do alimento. Vale salientar que algumas amostras de preparações à base de carne

Tabela 1. Conformidades* e respectivas médias das temperaturas, ao final da cocção, início e final da distribuição de preparações à base de carne em escolas municipais das regiões Leste, Oeste, Norte e Sul de Natal (RN), 2005.

\begin{tabular}{|c|c|c|c|c|c|c|c|c|}
\hline \multirow[t]{2}{*}{ Região administrativa } & \multirow[t]{2}{*}{ Escolas (n) } & \multirow[t]{2}{*}{ Conformidade } & \multicolumn{2}{|c|}{$\begin{array}{c}\mathrm{T}^{\circ} \mathrm{C} \text { final cocção } \\
>70,0^{\circ} \mathrm{C}\end{array}$} & \multicolumn{2}{|c|}{$\begin{array}{c}\mathrm{T}^{\circ} \mathrm{C} \text { início distribuição } \\
>60,0^{\circ} \mathrm{C}\end{array}$} & \multicolumn{2}{|c|}{$\begin{array}{c}\mathrm{T}^{\circ} \mathrm{C} \text { final distribuiçãc } \\
>60,0^{\circ} \mathrm{C}\end{array}$} \\
\hline & & & $M$ & DP & $M$ & DP & M & DP \\
\hline \multirow[t]{2}{*}{ Leste } & 3 & Não & 91,0 & 6,3 & 50,6 & 5,1 & 48,3 & 6,8 \\
\hline & 9 & Não & & & & & & \\
\hline \multirow[t]{2}{*}{ Oeste } & 1 & Sim & 92,4 & 4,9 & 54,2 & 6,8 & 49,0 & 10,6 \\
\hline & 10 & Não & & & & & & \\
\hline Norte & 1 & Sim & 93,6 & 4,4 & 51,1 & 8,1 & 49,0 & 9,3 \\
\hline Sul & 3 & Não & 91,5 & 5,2 & 48,1 & 4,7 & 46,5 & 4,0 \\
\hline
\end{tabular}

*Conformidade significa que a escola está dentro dos padrões em todas as fases avaliadas; M: média; DP: desvio-padrão. 
atingiram temperaturas superiores $a 0^{\circ} \mathrm{C}$ durante o início e/ou final da distribuição, em algumas escolas das diferentes regiões administrativas, e estes valores foram encontrados em amostras cujos alimentos foram servidos logo após o preparo.

A Figura 1 ilustra a distribuição percentual das temperaturas no início e no final da distribuição, em discordância à legislação, de acordo com a temperatura média de cada escola por região administrativa. Percebe-se que $100 \%$ das preparações nas Regiões Leste e Sul apresentaram temperaturas inadequadas no início e no final da distribuição, já que a legislação preconiza que os alimentos devem ser mantidos a temperaturas superiores a $60^{\circ} \mathrm{C}$.

Na Região Oeste 70\% das escolas apresentaram temperaturas em desacordo no início da distribuição e $90 \%$ no final desta etapa. $\mathrm{Na}$ Região Norte verificou-se que $91 \%$ das escolas apresentaram temperaturas abaixo de $60^{\circ} \mathrm{C}$ no início e $82 \%$ no final da distribuição.

A maior adequação no final da distribuição, na Região Norte, provavelmente, ocorreu em virtude da forma de armazenamento pós-cocção de algumas escolas, nas quais as preparações permaneciam em grandes panelas, o que permitia um maior resfriamento na superfície do alimento e, conseqüentemente, a porção que se

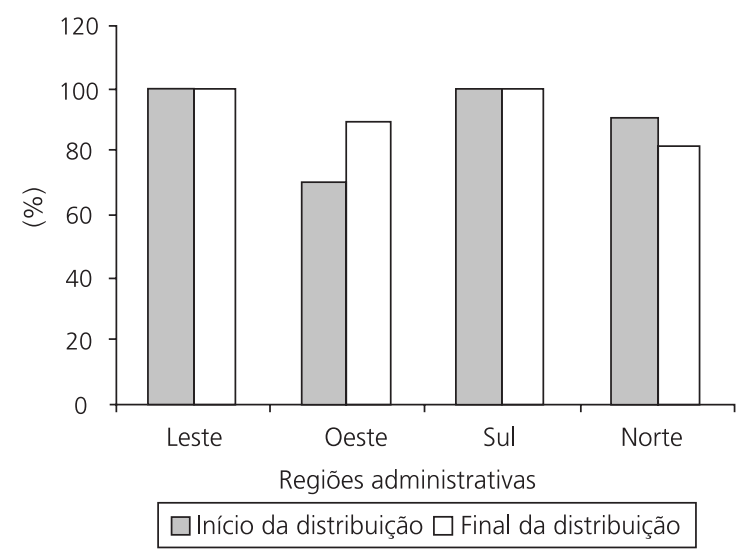

Figura 1. Percentual de não conformidade das temperaturas de distribuição de preparações à base de carne servidas em escolas municipais de Natal (RN), por região administrativa. 2005. encontrava no fundo do recipiente apresentava temperatura superior à inicial. Esse fato pode ter sido acentuado pela temperatura ambiente, cuja média anual, no município de Natal, em 2005, foi de $26,6^{\circ} \mathrm{C}^{18}$, e algumas escolas estudadas apresentavam temperaturas superiores a esta, devido à deficiência na ventilação.

Os resultados encontrados corroboram os de outros estudos. Silva et al. ${ }^{19}$, avaliando as condições higiênico-sanitárias da alimentação escolar em São Paulo, perceberam que os alimentos não submetidos a reaquecimento, no momento da distribuição, apresentavam temperaturas inferiores a $50^{\circ} \mathrm{C}$. Storck $\& \operatorname{Dias}^{20}$, ao monitorar as temperaturas de preparações quentes e frias em restaurantes do tipo self-service na zona urbana de Santa Maria (RS), encontraram 33,3\% das preparações quentes com temperatura inadequada. Chesca et al. ${ }^{21}$, avaliando temperaturas de distribuição de refeições frias e quentes em restaurantes de Uberaba (M G), observaram que $25,0 \%$ das preparações quentes estavam abaixo de $60^{\circ} \mathrm{C}$. Brugalli et al. ${ }^{22}$, ao investigarem perigos e pontos críticos de controle em um restaurante universitário da Universidade Federal do Rio Grande do Sul, constataram a exposição de alguns produtos, como peixe e frango fritos, a temperatura ambiente por períodos prolongados (cerca de 2 horas) e também que as carnes e guarnições encontravam-se a temperaturas inferiores $a 0^{\circ} \mathrm{C}$, nos do is balcões aquecidos.

A Tabela 2 mostra a média do tempo de espera das preparações à base de carne, ou seja, o tempo decorrido entre o final da cocção e o final da distribuição e também, o tempo de espera mínimo e máximo em cada região. Observou-se um tempo médio de espera de 57 minutos, 49 minutos, 66 minutos, 48 minutos, para as Regiões Leste, Oeste, Norte e Sul, respectivamente. 0 tempo máximo na Região Norte destaca-se com relação às demais. Vale salientar que, em apenas uma escola, a finalização de uma preparação ocorreu com demasiada antecedência ao consumo (151 minutos). 
Tabela 2. Média do tempo de espera das preparações à base de carne nas escolas municipais de Natal (RN), de acordo com as regiões administrativas. 2005.

\begin{tabular}{lcccc}
\hline \multirow{2}{*}{$\begin{array}{l}\text { Regiões } \\
\text { administrativas }\end{array}$} & Escolas (n) & \multicolumn{3}{c}{ Tempo de espera (minutos) } \\
\cline { 3 - 5 } & & M édia & M ínimo & Máximo \\
\hline Leste & 3 & 57 & 37 & 69 \\
Oeste & 10 & 49 & 26 & 83 \\
Norte & 11 & 66 & 33 & 151 \\
Sul & 3 & 49 & 33 & 64 \\
\hline Total & 27 & - & - & - \\
\hline
\end{tabular}

A Resolução RDC no 216, de 15/09/04, preconiza que os alimentos devem permanecer a temperaturas superiores a $60^{\circ} \mathrm{C}$ por, no máximo, 6 horas, ou seja, não devem permanecer a temperatura ambiente, como ocorreu em todas as escolas que fizeram parte do presente estudo. No estado de São Paulo vigora a portaria CVS-6-99, de 10/03/99, que preconiza que alimentos quentes podem ficar na distribuição ou espera, abaixo de $60^{\circ} \mathrm{C}$, por até 3 horas $^{23}$. No entanto, seria arriscado adotar esse parâmetro, tendo em vista a ausência de boas práticas e as condições precárias de higiene nas escolas municipais, observadas durante a coleta dos dados.

Desta forma, a faixa de tempo/temperatura encontrada nas preparações durante a distribuição pode ter propiciado o desenvolvimento bacteriano, pois a maioria delas encontrava-se com temperaturas abaixo do recomendado e o tempo de espera ultrapassou 48 minutos. Resultados diferentes foram obtidos por Vieira et al. ${ }^{17}$, que observaram que o tempo gasto entre o término do preparo e a distribuição de pratos prontos à base de carne nas escolas estaduais de Poços de Caldas foi de, aproximadamente, 30 minutos. Silva et al. ${ }^{24}$, avaliando os conhecimentos dos responsáveis pela alimentação escolar, por meio de um questionário, no que concerne ao tempo de espera, obtiveram que em $87,5 \%$ das unidades escolares, os alimentos são servidos imediatamente após o preparo.

O calor destrói parte ou toda a flora bacteriana, mas não possui efeito residual, isto é, finda sua ação, pode ocorrer recontaminação e/ou multiplicação. Por este motivo os produtos submetidos a um tratamento pelo calor devem ser consumidos de imediato ${ }^{21}$.

Durante o tempo de espera, foram observadas condições de exposição inadequadas das preparações à base de carne em todas as regiões administrativas. Algumas preparações permaneciam em recipientes tampados à temperatura ambiente até serem servidas.

Foi verificado que em algumas escolas preparações permaneciam sem tampa, o que pode facilitar a contaminação física e permitir que a temperatura do alimento decaia mais rapidamente. Observou-se, também, que $100 \%$ das escolas pesquisadas não apresentavam refeitórios e que a distribuição acontecia fora da cozinha, nas salas de aula, nos corredores e nas quadras esportivas, podendo comprometer a qualidade higiênico-sanitária dos alimentos.

Spinelli \& Canesqui ${ }^{25}$, avaliando o processo de implementação do programa descentralizado de alimentação escolar em Cuiabá, no período de 1993 a 1996, verificaram que a ausência de refeitórios foi problema em $100 \%$ das escolas cuiabanas estudadas. Dessa forma, as salas de aula, as quadras esportivas e outros locais eram improvisados como refeitórios, comprometendo as condições de higiene e dignidade requeridas à realização das refeições pelos alunos. Sousa et al. ${ }^{26}$, identificando os principais pontos críticos nas preparações com carne em uma Unidade de Alimentação e Nutrição hospitalar, observaram ineficácia dos equipamentos para manter temperaturas das carnes acima de $60^{\circ} \mathrm{C}$. Relataram, no entanto, que essas preparações apresentaram baixo níveis de coliformes totais, de origem fecal, e Escherichia coli, e atribuíram esse resultado, provavelmente, à pouca manipulação das preparações, que eram acondicionadas em cubas de inox e tampadas após a cocção.

É necessário garantir a segurança e a qualidade da alimentação escolar, na quantidade e regularidade necessária para 0 desenvolvimento dos alunos da rede pública de ensino do País. Deschamps et al. ${ }^{27}$ ressaltam que 0 controle do 
binômio tempo/temperatura é de fundamental importância para uma maior segurança alimentar durante o processo produtivo, evitando, assim, a proliferação de microorganismos nocivos à saúde. De acordo com Deus et al. ${ }^{28}$, a temperatura é um dos fatores que mais afeta a viabilidade e a multiplicação microbiana, portanto, o reaquecimento torna-se a última linha de defesa na prevenção de DTAs na produção de alimentos, porém, não foi observada esta prática em nenhuma das escolas estudadas.

\section{O N CLUSÃ O}

As temperaturas de cocção da alimentação escolar servida nas escolas pesquisadas apresentam-se adequadas, entretanto o binômio tempo/temperatura das preparações durante a distribuição, encontra-se fora dos padrões recomendados pela legislação, o que pode favorecer o crescimento e a multiplicação de bactérias, possibilitando a ocorrência de doenças transmitidas por alimentos. Logo, se faz necessária uma maior atenção ao fornecimento da alimentação escolar, principalmente no que se diz respeito às preparações à base de carne, pois estas irregularidades poderão comprometer a segurança do alimento e, conseqüentemente, a saúde das crianças beneficiadas pelo Programa Nacional de Alimentação Escolar.

Desta forma, os resultados obtidos no presente estudo permitem sugerir:

- a presença de responsáveis técnicos qualificados para supervisão e orientação periódica das atividades desenvolvidas nas Unidades de Alimentação e Nutrição das escolas municipais;

- locais adequados para distribuição dos alimentos preparados (refeitórios);

- a aquisição de equipamentos que mantenham os alimentos preparados protegidos de contaminação e em temperatura correta, já que não é possível, em todas as escolas, servir logo após o preparo.

\section{A GRA DECIMENTOS}

Ao Departamento de Nutrição e à Secretaria Municipal de Educação da Prefeitura de Natal, pelo apoio à execução do trabalho.

\section{OLA BORA DORES}

M.S. ROSA participou na coleta de dados, na tabulação, na análise dos dados e na redação do artigo. S.R.F. NEG REIROS participou na coleta de dados e na redação do artigo. L.M .J. SEABRA participou na análise e interpretação dos dados. T.L.M. STAM FORD participou na análise, interpretação dos dados e na redação do artigo.

\section{REFERÊ N CIAS}

1. Brasil. Fundo Nacional de Desenvolvimento da Educação. Programa Nacional de Alimentação Escolar: É hora da merenda! Brasília: Ministério da Educação; 2001.

2. Brasil. Ministério da Educação. Resolução/FNDE/ CD/n. 32 de 10 de agosto de 2006 [acesso em 10 set 2007]. Disponível em: <tp://ftp.fnde.gov.br/ web/resolucoes_2006/res032_10082006.pdf >.

3. Costa EQ, Ribeiro VMB, Ribeiro ECO. Programa de alimentação escolar: espaço de aprendizagem e produção de conhecimento. Rev Nutr. 2001; 14(3): 225-9.

4. Pipitone $M A P$, Ometto $A M H$, Silva $M V$, Sturion $G L$, Furtuoso M CO, Oetterer M. Atuação dos conselhos municipais de alimentação escolar na gestão do programa nacional de alimentação escolar. Rev Nutr. 2003; 16(2):143-54.

5. Brasil. Ministério da Educação. Resolução /FNDE/ $\mathrm{CD} / \mathrm{no} 38$ de 23 de agosto de 2004 [acesso em 10 set 2007]. Disponível em: <ftp://ftp.fnde.gov.br/ web/ resolucoes_2004/res038_23082004.pdf>.

6. Secretaria de Estado da Educação. Programa: alimentação escolar do Distrito Federal-PEAE/DF. Brasília; 2004 [acesso em 10 set 2007]. Disponível em: <http://www.se.df.gov.br/programasprojetos/ AlimentacaoEscolanoDFPrograma.pdf>.

7. Phipippi ST. Nutrição e técnica dietética. São Paulo: M anole; 2003.

8. Franco BDGM, Landgraf M. Microbiologia dos alimentos. São Paulo: Atheneu; 2005.

9. World Health Organization. Five keys to safer food manual [cited 2007 Sept 10]. Available from: 〈http:// 
www.who.int/foodsafety/publications/consumer/ manual_keys.pdf>.

10. Silva Jr EA. M anual de controle higiênico sanitário em serviços de alimentação. 6a. ed. São Paulo: Livraria Varela; 2005.

11. Hobbs BC, Roberts D. Toxinfecções e controle higiênico-sanitário de alimentos. São Paulo: Livraria Varela; 1999.

12. Brasil. M inistério da Saúde. Resolução RDC $n 0216$, de 15 de setembro de 2004 [acesso em 2 nov 2004]. Disponível em: <http://e-legis.anvisa.gov.br /leisref/public/show Act.php?id=12546\&w ord=>.

13. Scheaffer RL, Mendenhall W, Ott L. Elementary survey sampling. 5th ed. Boston: Duxbury Press; 1996.

14. Statsoft Inc. [CD-ROM] Statistica: data analysis software system, version 6. 2001.

15. Oliveira ACB, Germano PML, Germano MIS. Avaliação dos alimentos cárneos servidos no programa de alimentação escolar de um município da grande são Paulo: ênfase nos aspectos de tempo e temperatura. Hig Aliment. 2004; 18(124):24-9.

16. Fortuna JL. Aspectos higiênico-sanitário no preparo de carne bovina servida em refeições escolares de instituições escolares municipais e estaduais, no Estado do Rio de Janeiro. Hig Aliment. 2002; 16(95):23-33.

17. Vieira CRN, Silva RR, Martino HSD, Chavasco JK. Qualidade microbiológica da merenda escolar servida nas escolas estaduais de Poços de Caldas, MG. Hig Aliment. 2005; 19(128):90-4.

18. Prefeitura de Natal. Secretaria Municipal de Meio Ambiente e Urbanismo. Departamento de Informação, Pesquisa e Estatística. Anuário Natal 2005. Natal (Brasil): Prefeitura de Natal; 2005.

19. Silva C, Germano MIS, Germano PML. Avaliação das condições higiênico-sanitárias da merenda escolar. Hig Aliment. 2000; 14(71):24-31.

20. Storck CR, Dias MAMF. Monitoramento da temperatura de preparações quentes e frias em restaurantes self-service na zona urbana de Santa Maria. Rev Nutr Pauta. 2003; 59:30-4.

21. Chesca AC, Caetano AM, Leite APC, Polveiro AM, Terra AD, Lyra FS, et al. Avaliação das temperaturas de pistas frias e pistas quentes em restaurantes da cidade de Uberaba, MG. Hig Aliment. 2001; 15(87):38-43.

22. Brugalli A, Pinto JM, Tondo EC. Análise de perigos e pontos críticos de controle para garantir a segurança alimentar em restaurante da Universidade Federal do Rio Grande do Sul. Hig Aliment. 2000; 14(72):53-9.

23. São Paulo. Centro de Vigilância Sanitária. Portaria CVS 6 de 10 de março de 1999 [acesso em 10 set 2007]. Disponível em: <http://www.cvs.saude.sp. gov. br/dow nload. asp? tipo $=$ zip \&arquivo $=99$ pcvs6.zip>.

24. Silva C, Germano MIS, Germano PML. Conhecimento dos manipuladores da merenda escolar em escolas da rede estadual de ensino em São Paulo-SP. Hig Aliment. 2003; 17(113):46-51.

25. Spinelli MAS, Canesqui AM. Descentralização do Programa de Alimentação Escolar em Cuiabá: 1993-1996. Rev Nutr. 2004; 17(2):151-65.

26. Sousa AA, Salles RK, Mormello P, Tosin I, Felipe MR. Identificação de pontos críticos em uma unidade de alimentação e nutrição hospitalar: subsídios para implantação do sistema HACCP. Hig Aliment. 2001; 15(84):25-43.

27. Deschamps C, Freygang J, Bramorski A, Tommasi D, Garcia GF. Avaliação higiênico-sanitária de cozinhas industriais instaladas no município de Blumenal-SC. Hig Aliment. 2003; 17(112):12-5.

28. Deus MB, Holland N, M oraes CM M , Stamford TLM . Microrganismos patogênicos e temperaturas de exposição de carne bovina servida em restaurantes self-service da cidade de Natal (RN), Brasil. Revisa. 2005; 1(4):237-45.

Recebido em: 24/8/2006

Versão final reapresentada em: 27/9/2007 Aprovado em: 22/10/2007 